\title{
Akute Lahmheit in der Außenpraxis
}

\section{Sorgfaltspflicht und diagnostische Möglichkeiten}

\author{
Maria Fugazzola
}

Die akute, hochgradige Lahmheit des Pferdes stellt für den Tierarzt in der Außenpraxis oft eine große Herausforderung dar. Mit dem Wissen, dass aus einer möglichen Fissur doch noch eine Fraktur resultieren kann, bleibt die Lahmheitsuntersuchung oft auf die klinische Untersuchung beschränkt. Ziel dieses Artikels ist es zu erläutern, welche Anhaltspunkte vor Ort eine Fissur eher unwahrscheinlich erscheinen lassen bzw. ab wann ein Pferd besser überwiesen werden sollte. Dabei sollen die wichtigsten Ursachen sowie ihre Symptome für akute, hochgradige Lahmheiten veranschaulicht und darauf hingewiesen werden, was in der Außenpraxis möglich ist und was gerade nicht.

\section{Einleitung}

Bei einer akuten, hochgradigen Lahmheit bleibt die Lahmheitsuntersuchung oft auf die klinische Untersuchung beschränkt, dies vor dem Hintergrund, dass aus einer möglichen Fissur doch noch eine Fraktur resultieren kann. Damit verbleiben gerade bei der Erstuntersuchung nicht selten große Zweifel im Hinblick auf die genaue Diagnose. Die Angst, wichtige Symptome zu übersehen oder gar eine Verschlimmerung durch weiterführende Diagnostik herbei zu führen ist nicht unbegründet: Wie im humanmedizinischen Bereich kommt es dann zu einer Beweislastumkehr, wenn dem Tierarzt grobe Behandlungsfehler nachgewiesen werden können. So urteilte der Bundesgerichtshof in Karlsruhe am 10.05.2016, dass der erstuntersuchende Tierarzt einer vorberichtlich bekannten Schlagverletzung durch mangelnde Abklärung einer Fissur maßgeblich dazu beigetragen habe, dass das Bein des Pferdes letztlich frakturiert sei. Er wurde hierfür in Haftung genommen.

\section{Die Bedeutung der klinischen Untersuchung}

Die akut auftretende Lahmheit kann verschiedenste Ursachen haben. Für viele ambulante Pferdepraxen gehören moderne bildgebenden Verfahren wie etwa ein digitales Röntgensystem mittlerweile zur Standardausstattung. Damit gerät allerdings aufgrund der vermeintlich schnelleren Diagnostik die klinische Untersuchung oft ins Hintertreffen. Mit diesem Bewusstsein jedoch sollte sie wieder zurück an erste Stelle gesetzt werden.

Die klinische Untersuchung beginnt mit der Erhebung der Anamnese und mündet in eine gründliche Adspektion und Palpation. Diese können schon viele Hinweise auf die Diagnose geben. Dabei sind die Beobachtung des Bewegungsmusters des Patienten sowie ein strukturiertes Vorgehen bei der Untersuchung von besonderer Wichtigkeit.

\section{Traumaursache bekannt}

Ist die Ursache des Traumas bekannt, bleiben Adspektion und Palpation oft die einzigen diagnostischen Maßnahmen. Diagnostische Anästhesien sind in einem solchen Fall nämlich kontraindiziert. Eine sorgfältige Palpation der gesamten Gliedmaße und eine Stabilisierung durch einen entsprechenden Verband bei Frakturverdacht sind bei dem Erstbesuch meistens die einzig sinnvollen Vorgehensweisen.

\section{Traumaursache unbekannt}

Ist die Ursache der Lahmheit dagegen unbekannt, beginnt die Untersuchung gleichwohl mit Erhebung der Anamnese, Adspektion und Palpation.

Zu den wichtigsten Befunden gehören:

- Weichteilschwellungen

- Füllung von Synovialstrukturen

- Druckdolenz und/oder

- Flexions- und Torsionsschmerz

- vermehrte Wärme
Kann hiernach die Ursache der Lahmheit nicht ermittelt werden, sollte man sich zunächst dem Hufbereich zuwenden. Hierbei wird dem Vorhandensein eines Wendeschmerzes, der einseitig oder beidseitig auftreten kann, besondere Aufmerksamkeit geschenkt. Diese Symptomatik gilt als typisch für unilaterale Hufproblematiken und ist z.B. charakteristisch für Hufbeinastfrakturen.

\section{Häufige Lahmheitsursachen}

Als häufigste Ursachen für akute, hochgradige Lahmheiten, die ihre Ursache im Huf haben, gelten Sohlenlederhautprellungen bzw. -quetschungen, Nageltritte, Hufabszesse, Hufrehe, akute Schäden der Weichteilstrukturen (oft der tiefen Beugesehne) sowie Hufbeinfrakturen. Andere Lahmheitsursachen wie das Hufrollensyndrom (Podotrochleose) oder eine Hufgelenksarthrose zeigen dagegen tendenziell eher einen chronischen Verlauf.

Die meisten genannten Erkrankungen sind i.d.R. in der Außenpraxis gut diagnostizier- und therapierbar ( $\bullet$ Tab. 1). Im Folgenden sollen sie nun genauer erläutert werden.

\section{Huflederhautprellungen und -quetschungen}

Durch ein Trauma der Sohle kommt es zur Einblutung in die Sohlenlederhaut und führt damit zu einem erhöhten Druck zwischen Lederhaut und Hornsohle. Die häufigste Lokalisation für solche Traumata ist der Bereich zwischen Eckstrebe und Hufwand. Auch kann ein zu stark nach innen gebogenes Eisen oder ein verrutschtes Eisen zu einer solchen Quetschung der Lederhaut führen. Traumata der Sohlenlederhaut im Bereich um die Hufbeinspitze sind dagegen häufig bei Pferden mit niedrigen Trachten zu finden. Hier hat die Sohle im Bereich der Hufbeinspitze häufiger 
Tab. 1 Welche Ursachen für akute, hochgradige Lahmheiten können in der Außenpraxis diagnostiziert und behandelt werden und welche sollten in eine Klinik überwiesen werden?

\begin{tabular}{|lcc|}
\hline & \multicolumn{2}{c}{ Diagnostik und Therapie in } \\
\hline Lahmheitsursache & Außenpraxis & Klinik \\
\hline Huflederhautprellung/-quetschung & + & $(+)$ \\
\hline Hufabszess & + & + \\
\hline Nageltritt & - & + \\
\hline Hufrehe & $(+)$ & $+(+)$ \\
\hline Hufbeinfraktur & + & +
\end{tabular}

Kontakt zum Boden und kann dadurch leicht gequetscht werden. Vorberichtlich geht diesen Verletzungen oft ein Reiten auf steinigem Boden voraus.

Bei einer Huflederhautprellung verschlimmert das Abnehmen des Eisens die Lahmheit oftmals.

Die Pulsation der Mittelfußarterie ist deutlich fühlbar bis stark pochend beschrieben und die Hufzangenprobe meistens auf der gesamten Sohlenfläche positiv. Häufig kann man beim Beschneiden des Verfallshorns über der druckdolenten Stelle eine Verfärbung der Hufsohle erkennen $(\triangleright$ Abb. 1).

Die tiefe Palmarnervenanästhesie fällt häufig positiv aus. Dagegen zeigt die radiologische Untersuchung nur in seltenen Fällen eine radioluszente Flüssigkeitslinie zwischen Hufbein und Sohle.

\section{Therapie}

Die Therapie der Wahl sind Entzündungshemmer, z.B.

- Phenylbutazon: $2,2 \mathrm{mg} / \mathrm{kg}$ KGW $2 \times$ tgl. (cave! Lebensmittelstatus beachten) und,

falls erforderlich, das Beheben der Ursache.

Es empfiehlt sich, einen Hufpolsterverband an die betroffene Gliedmaße anzubringen, um die Zeit zum nötigen Beschlag zu überbrücken und die geprellte Sohle zu schützen. Anschließend sollte so bald wie möglich ein adäquater Beschlag mit Sohleneinlage angebracht werden, wobei der Bereich über der verletzten Region der Sohle vorsichtig ausgeschnitten wird, sodass dieser Teil möglichst nicht gewicht- tragend ist. Bei der Wahl des Beschlags sollte vor allem auf die Position des Eisens zur Hufwand geachtet werden, um Druck auf die Sohle vollständig zu vermeiden.

\section{Prognose}

Bei einer adäquaten Therapie der Huflederhautprellung oder -quetschung ist die Prognose sehr günstig.

\section{Hufabszess}

Der Hufabszess (Pododermatitis septica) gehört zu den am häufigsten vorkommenden Ursachen für akute, mitunter hochgradige Lahmheiten beim Pferd. In der Regel resultiert er aus einem Sohlentrauma oder einer penetrierenden Wunde an der weißen Linie bzw. an einem Nagelloch.

Die Lahmheit ist typischerweise hoch- bis höchstgradig. Eine begleitende Schwel-

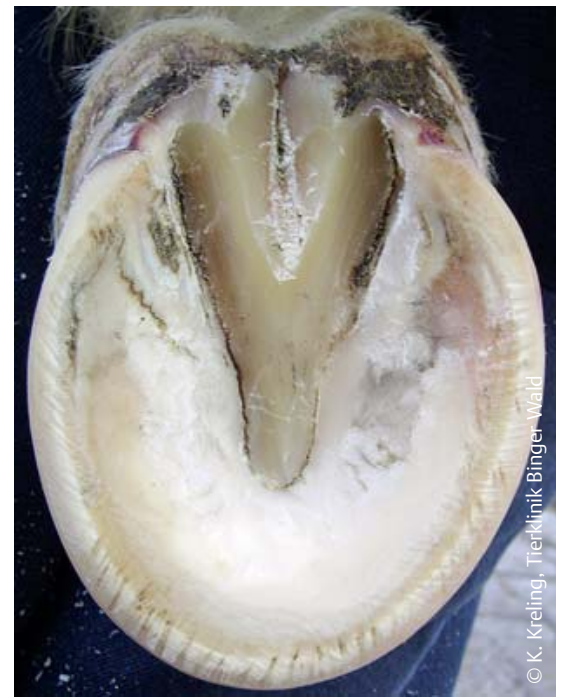

Abb. 1 Verfärbung des Horns nach Entfernung des Zerfallshorns über der zangendruckdolenten Stelle im Eckstrebenbereich. 


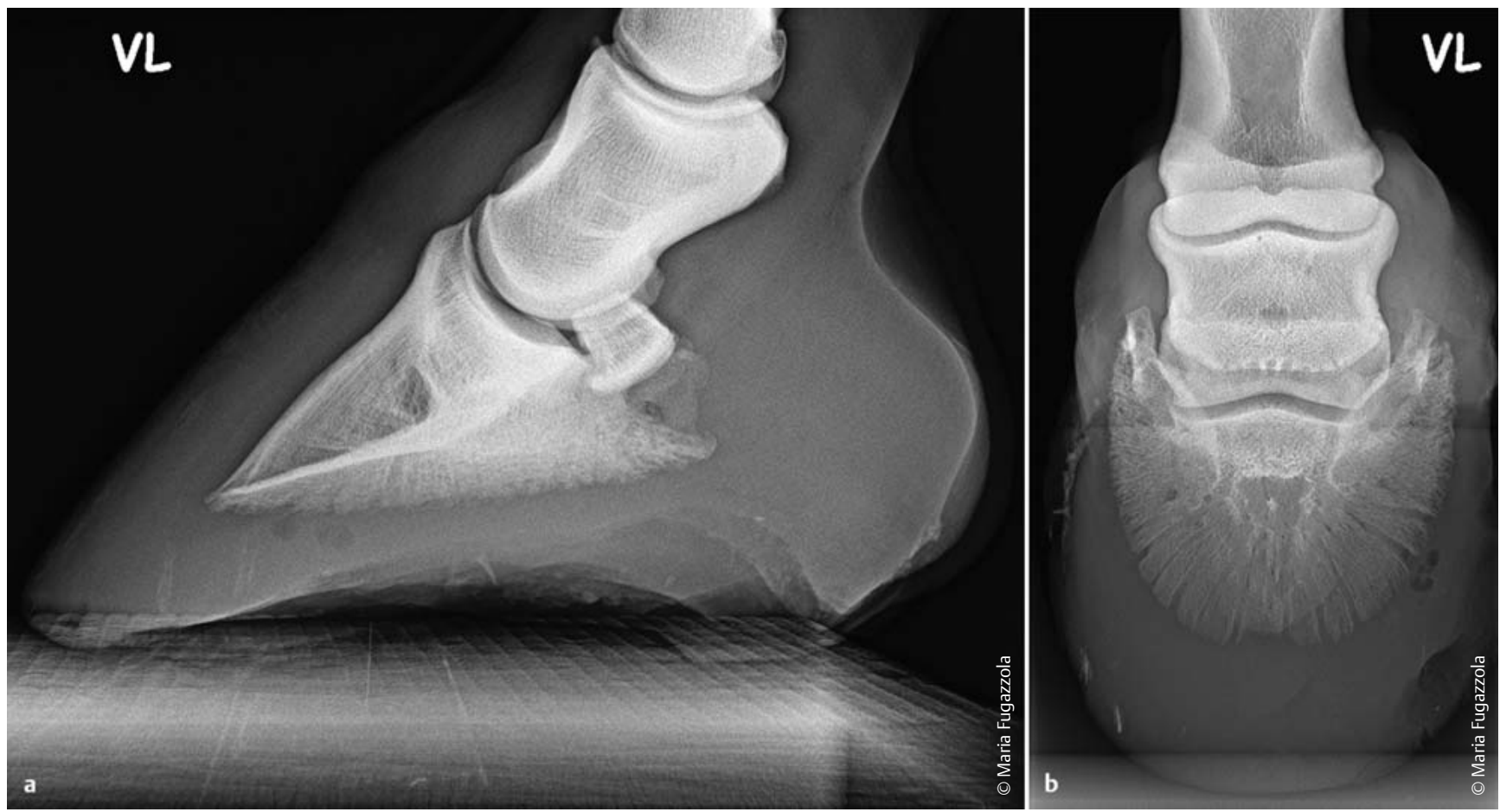

Abb. 2 a und b Röntgenaufnahmen der Zehe. a Standardaufnahme seitlich $\left(90^{\circ}\right)$ : Lufteinschlüsse an der Hufbeinspitze. b Oxspring-Aufnahme: Lufteinschlüsse lateral des Hufbeinrands - diese weisen auf die Lokalisation des Hufabszesses hin.

lung der distalen Gliedmaße inklusive Füllung der Fesselbeugesehnenscheide lässt initial fälschlicherweise eine Weichteilproblematik vermuten, die jedoch durch eine gründliche klinische Untersuchung oftmals schnell wieder verworfen wird.

Symptomatisch stehen eine verstärkte Pulsation der Mittelfußarterie und oft eine vermehrt warme Hufkapsel im Vordergrund. In den meisten Fällen lässt sich durch sorgfältiges Arbeiten mit der Hufzange eine bestimmte druckempfindliche Region herausstellen. Je nach Lokalisation können die Pferde auf der einen Hand stärkeren Wendeschmerz als auf der anderen zeigen.

Bei einem Verdacht auf einen Hufabszess sollte immer das Eisen entfernt und die Sohle behutsam nachgeschnitten werden.

Dabei ist das gesunde Horn möglichst zu schonen, um den Schaden nicht noch zu erweitern. Bei Pferden mit sehr hartem Horn kann ein für 24 Stunden angelegter warmer Angussverband (z.B. mit warmem Seifenwasser) hilfreich sein, um das Horn über dem Abszess zu erweichen und beim Nachschneiden besser zugänglich zu machen.

\section{Röntgen}

Manchmal kann ein Röntgenbild zusätzlich wichtige Informationen liefern, vor allem, wenn der Verdacht auf Beteiligung tieferer Strukturen oder auf eine großflächige Unterminierung besteht. Es empfiehlt sich, die Anfertigung einer seitlichen Zehen- sowie einer Oxspring-Aufnahme $(\checkmark$ Abb. 2 a und $\mathbf{b})$.

\section{Therapie}

Bei der Eröffnung des Abszesses fließt zumeist ein dunkles, stinkendes Material ab. Mittels Zangendruck über der gesamten Sohle und um die Abszessöffnung herum sollte festgestellt werden, wie weit die Sohle unterminiert ist und ob deshalb ein Vergrößern der Öffnung indiziert ist. Wichtig ist, dass das Ausheilen des Abszesses kontrolliert wird und es ggf. eines weiteren Nachschneidens bedarf, da gerade größere Hufabszesse öfter gekammert sind und einen größeren Teil der Sohle unterminieren können. Sie ziehen mitunter bis zum Kronrand.

Muss dagegen großflächig Material abgetragen werden, bei dem die Lederhaut vorfallen kann, empfiehlt sich eine Überweisung an die Klinik, um eine Lokalanästhesie mit Anlegen eines Blutstaus vorzuneh- men. Das Wundbett ist entsprechend $\mathrm{zu}$ versorgen, um mittels Gegendruck einen Lederhautvorfall zu vermeiden.

Die Gefahr von nicht vollständig ausgeheilten Hufabszessen liegt vor allem darin, zu Hornsäulen mit chronischen Lahmheiten möglicherweise mit Beteiligung der knöchernen Strukturen (genauer: des Hufbeins) und der Entstehung einer Osteomyelitis zu führen ( $\mathbf{A b b}$. 3). Ein Hufabszess darf deshalb nicht unterschätzt oder gar als Bagatelle abgetan werden.

Bricht der Abszess am Kronrand auf, ist unbedingt Abfluss nach unten zu schaffen und eine Lavage von der proximalen zur distalen Öffnung indiziert. Um eine Kontaminierung des Krongelenks zu vermeiden, sollte ein Debridement am Kronsaum so wenig invasiv wie möglich gehalten werden. Nach adäquater Eröffnung des Abszesses sollte der Huf einige Tage mit Angussverband und systemischen Entzündungshemmern behandelt werden. Trockene Verbänden schließen die Therapie ab. Eine Antibiotikatherapie ist in den meisten Fällen nicht notwendig. 


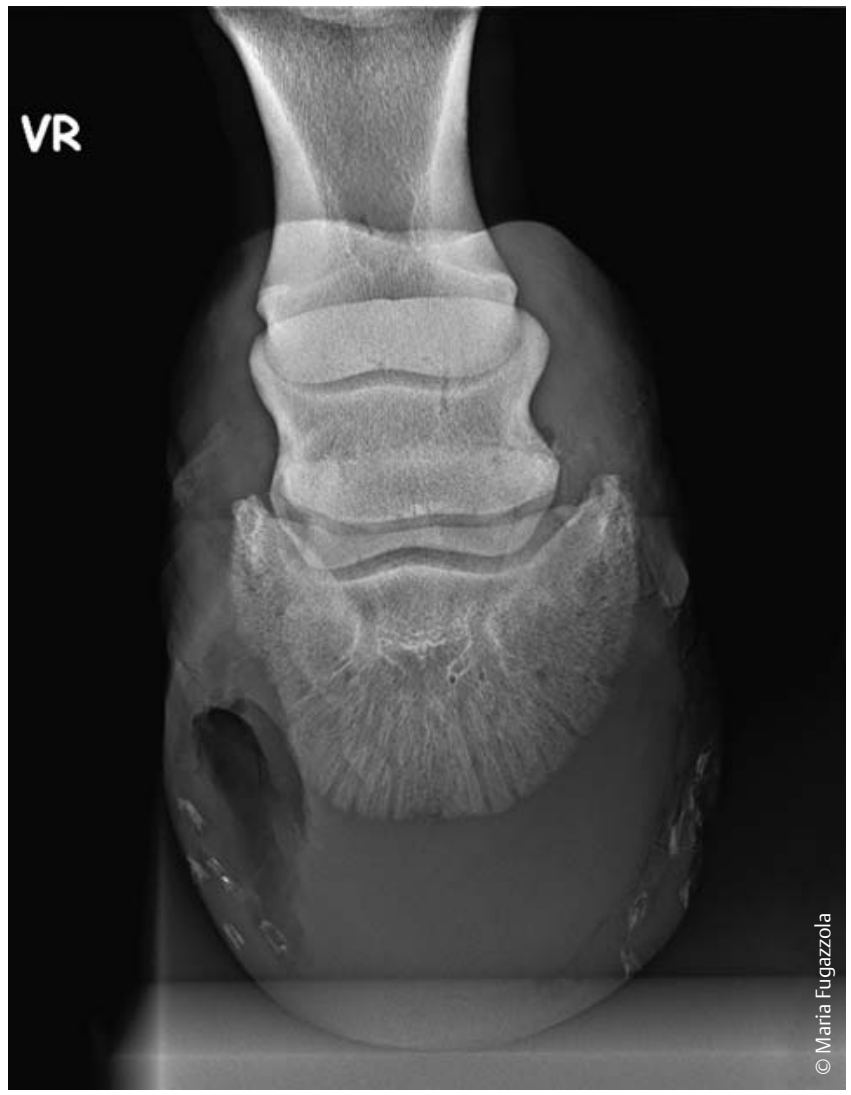

Prognose

Bei einem einfachen, direkt unter der Sohlenfläche des Horns befindlichen Abszess ist die Prognose sehr günstig. Sie verschlechtert sich jedoch bei Chronizität und wenn tiefere Strukturen betroffen sind.

\section{Nageltritt}

Prinzipiell sollten alle Stichwunden beim Pferd ernst genommen werden. Im Bereich von Hufsohle und Strahl bedürfen diese jedoch unserer besonderen Aufmerksamkeit. Sie sind extrem gefährlich und sollten immer als Notfall betrachtet werden. Die Stichwunden bergen nämlich die Gefahr, dass tiefer liegende Strukturen wie die Bursa podotrochlearis, die tiefe Beugesehne, die Fesselbeugesehnenscheide oder gar das Hufbein betroffen sein können. Wenn der Fremdkörper derlei Strukturen verletzt hat, sind die Pferde in den meisten Fällen hochgradig lahm.

Die Abwesenheit klinischer Auffälligkeiten im Bereich der proximalen Gliedmaße bei gleichzeitiger Anwesenheit einer verstärkten Pulsation der Mittelfußarterie führt schnell zum vermutlichen Sitz der Lahmheit.

Abb. 3 Röntgenologische Darstellung des rechten Vorderhufs in einer Oxspring-Aufnahme. An der lateralen Eckstrebe wurde ein chronischer Hufabszess ausgeschnitten (röntgenologische Aufhellung sichtbar). Der laterale Aspekt des Hufbeinrands zeigt bereits eine osteolytische Knochenresorption.

\section{Weiterführende Diagnostik}

Muss eine Penetration durch einen Fremdkörper befürchtet werden, sollte der eingedrungene Gegenstand - soweit möglich - umgehend entfernt werden, um weiteren Schaden zu vermeiden. Es besteht ein hohes Risiko, dass der Fremdkörper tiefer in die Sohle eindringt und hierbei synoviale Strukturen und die tiefe Beugesehne schädigt. Nach dieser Erstversorgung sollte die Gliedmaße mit einem Polsterverband versehen und das Pferd zeitnah in eine Klinik überwiesen werden. Die Zeit spielt hierbei eine wichtige Rolle, da sich durch das verlängerte Warten, die Propagation der Verletzung und die Kontamination synovialer Strukturen die Prognose verschlechtert. Antibiose und Tetanus-Antitoxin sollten vor der Überweisung schon verabreicht werden.

Bei Ankunft in der Klinik wird die Sohle unter Leitungsanästhesie ausgeschnitten, um nach dem Fremdkörpereingang zu suchen. Dieser kann dann nach steriler Vorbereitung sondiert und der Huf zeitgleich geröntgt werden, um die genaue Richtung des Stichkanals festzustellen. Aufgrund des Risikos, bei Abwehrreaktionen eine zusätzliche Verletzung zu verursachen, wird jedoch das sterile Füllen der Synovialstrukturen mit Kontrastmittel (der Bursa podotrochlearis und der Fesselbeugesehnenscheide) durch einen proximalen Zugang bevorzugt. Hiernach wird abermals ein Röntgenbild angefertigt, wobei das eventuelle Austreten der Kontrastflüssigkeit durch den Stichkanal sichtbar wird.

Die genaueste präoperative Prognose und Einschätzung des Schadens ist jedoch nur mittels MRT möglich, da hierbei das Ausmaß der Verletzung aller Weichteilstrukturen am besten dargestellt wird.

\section{Therapie}

Anschließend erfolgt die Therapie in Form einer Operation in Vollnarkose, bei der die betroffenen Synovialstrukturen gespült werden und die Stichwunde debridiert wird.

\section{Prognose}

Die Prognose hängt davon ab, welche und wie schwer die Strukturen geschädigt sind.

\section{Hufrehe}

Die Diagnose einer Hufrehe (Laminitis aseptica) wird meist aufgrund der Adspektion und der klinischen Symptomatik gestellt. Wenn es sich um einen akuten Schub innerhalb einer chronischen Hufrehe handelt, zeigt der Huf bei der Adspektion eine typische Ringbildung des Hornes, die sich durch die wiederholten Wachstumsstörungen während der akuten Reheschübe bildet. Auch eine verbreiterte weiße Linie der Sohle gehört zu den adspektorischen Merkmalen einer Rehe mit längerer Vorgeschichte.

Wenn nur die Vordergliedmaße betroffen ist, zeigt sich das typische Bild eines Pferdes mit Gewichtsverlagerung nach hinten bei vorgestreckten Vordergliedmaßen. In manchen Fällen sind auch die Hintergliedmaßen betroffen. Hierbei gestaltet sich die Abgrenzung von anderen Differenzialdiagnosen, wie z.B. durch andere Ursachen bedingte Schmerzhaftigkeiten der Sohle schwieriger. Pferde mit Hinterhandrehe bewegen sich nur äußerst widerwillig und fallen durch einen sehr steifen Gang auf. 


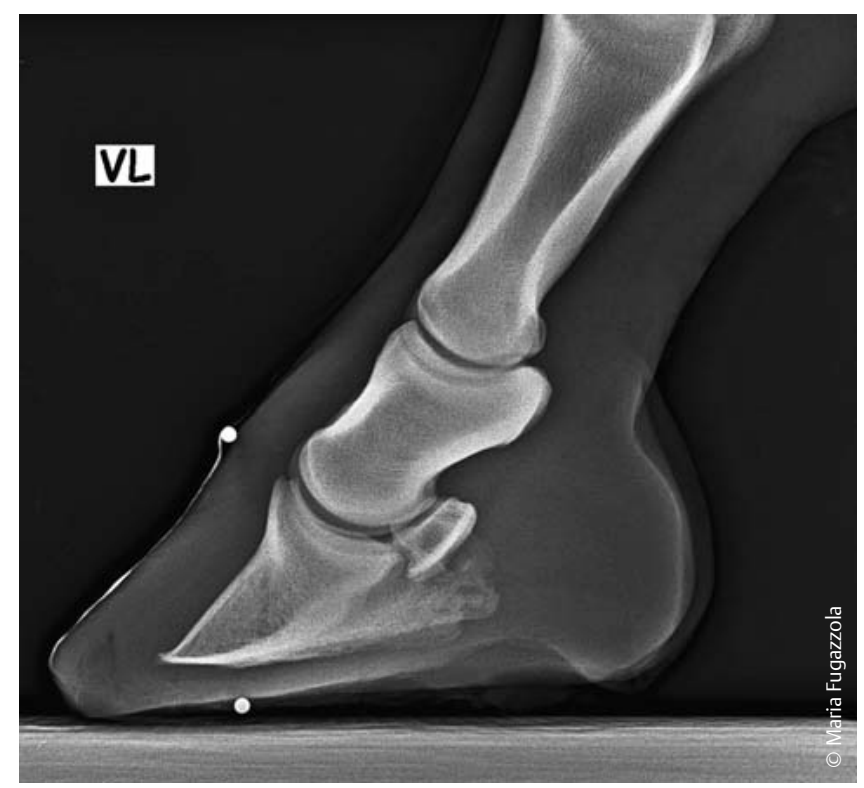

Abb. $490^{\circ}$-Aufnahme eines chronischen Rehehufs mit Markern: geringgradige Rotation des Hufbeins in der Hufkapsel und leichte Krempenbildung der Hufbeinspitze. An der Hufspitze sind geringgradige Lufteinschlüsse zwischen Hufwand und Lederhaut sichtbar.

Ein wichtiges Symptom ist eine beidseitige, stark pochende Pulsation der Mittelfußarterie. Diese ist durch die drastische Veränderung der Blutzirkulation des Hufes zu erklären, insbesondere durch die gestörte kapilläre Durchblutung der epidermalen Lamellen und den Bypass über dilatierte arteriovenöse Shunts. Das Abtasten der Sohle mit der Hufzange zeigt besonders an der Hufspitze eine deutliche Reaktion. In der akuten Phase ist der Huf stark erwärmt und eine Perkussion der Hufkapsel, besonders aber an der Hufspitze, erzeugt schmerzhafte Abwehrreaktionen.

Eine sorgfältige Palpation des Kronrands kann seine Einsenkung ergeben, was sich durch das Absenken des Hufbeins in der Hufkapsel erklären lässt. Der Effekt diagnostischer Anästhesien ist variabel und hängt nicht immer mit dem Grad der Rehe und der Lahmheit zusammen, weshalb diese nicht als ausschlaggebendes diagnostisches Mittel gebraucht werden sollten.

\section{Röntgen}

Die Durchführung standardisierter Röntgenaufnahmen der Zehe stellt die Basis für Therapie und Prognose dar. Während eine Rotation des Hufbeins oftmals erfolgreich therapiert werden kann, hat die Senkung des Hufbeins eine deutlich schlechtere Prognose. Die Röntgenbilder sind auch hilfreich, um ein akutes von einem eher chronischen Geschehen zu unterscheiden.

Bei den Röntgenaufnahmen der Zehe ist auf eine abnormale Breite der weißen Linie und die dorsale Ausziehung des Hufbeins (sog. Hutkrempenbildung) zu achten.

Die orthograde lateromediale Aufnahme ( $\triangleright$ Abb.4) ist wichtig, um die Position des Hufbeins in der Kapsel zu erfassen. Eine dorsopalmare/-plantare Aufnahme kann lateromediale Imbalanzen bei chronischer Hufrehe feststellen. Bei starken Veränderungen 


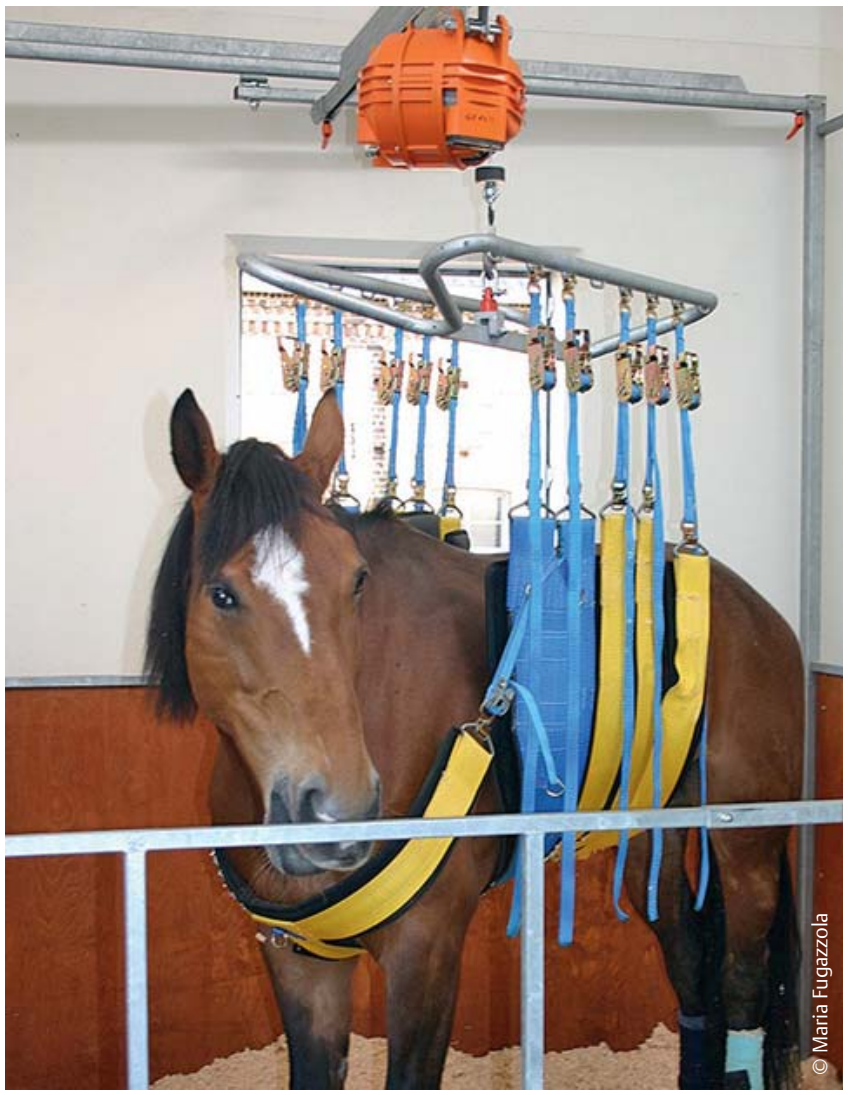

Abb. 5 Pferd im Swinglifter (Fa. Puhl GmbH).

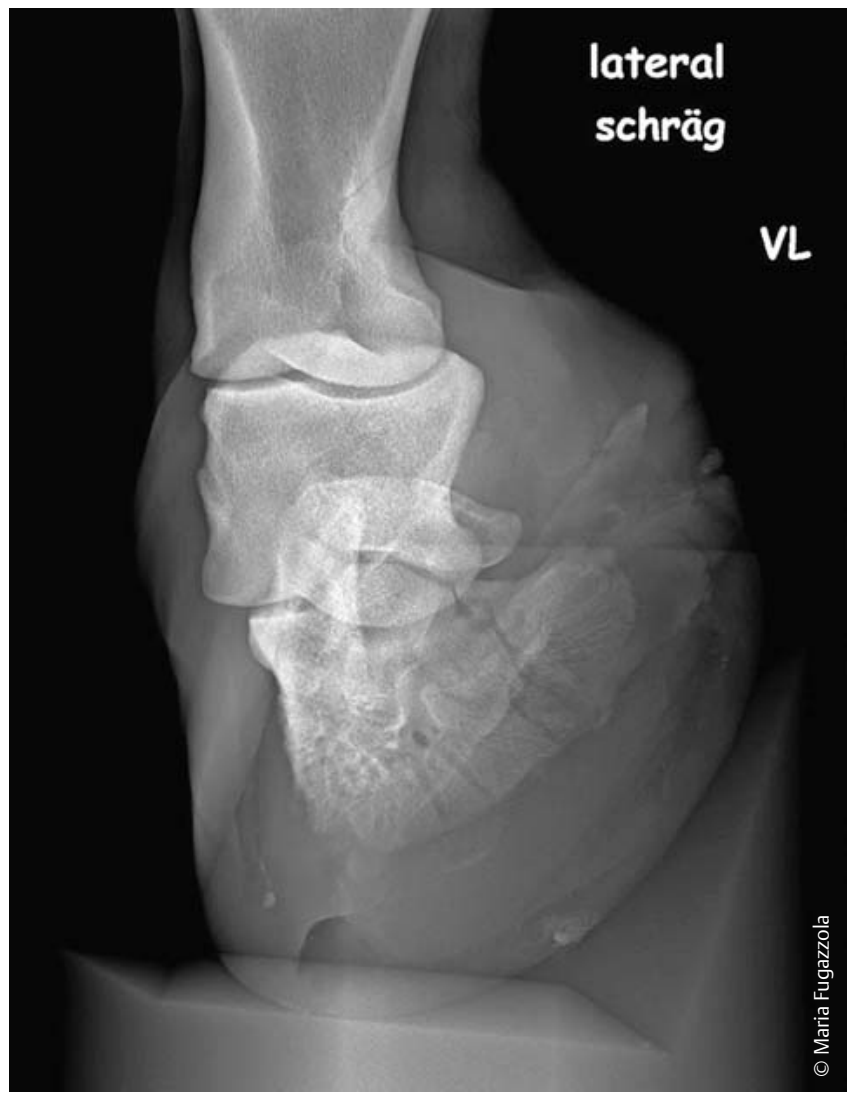

Abb. 6 Oxspring-Aufnahme, Zehe vorne links, $45^{\circ}$ : eine röntgendurchlässige Linie zieht im Hufbein von proximal nach distal - eine Hufbeinastfraktur mit artikulärer Komponente. der Hornkapsel sollte der Huf vor dem Röntgen bearbeitet werden, um eine unregelmäßig lange Zehe nicht mit einer Rotation des Hufbeins zu verwechseln.

Das Standardisieren der Röntgenaufnahmen beinhaltet das Setzen von röntgendichten Markern am Kronrand (Ansatz der letzten Haare), der dorsalen Hufwand und der Spitze des Strahls an der Sohle. Somit können die Dicke der Sohle, der Abstand des Proc. extensorius vom Kronrand (Rehestrecke), die Hufwanddicke und eine Rotation gemessen werden. Die Aufnahme sollte $90^{\circ}$ zur Gliedmaße, mit dem Fokus zwischen Hufspitze und Ballen, circa $2 \mathrm{~cm}$ distal des Kronrands angefertigt werden.

\section{Therapie}

Aktuell gibt es keine einheitliche Therapie, und nur das Ausmaß des Schadens selbst hat einen Einfluss auf die Prognose. Da eine Hufrehe oft die Konsequenz einer vorangehenden Erkrankung ist, die unabhängig von der betroffenen Gliedmaße im Körper stattfindet (Endotoxämie, Nachgeburtsverhalten, Erkrankung der kontrala- teralen Gliedmaße usw.), sollte zeitgleich zur spezifischen Rehetherapie der Auslöser schnellstmöglich behoben werden.

Es konnte gezeigt werden, dass in der Anfangsphase der Rehe eine Kühlung der Hufe mit Eiswasser zu einem Sistieren der Symptomatik geführt hat. Erklärt wird dies dadurch, dass die Kältetherapie in der akuten Phase zu einer Gefäßkonstriktion führt und somit die Triggerfaktoren in den Dermallamellen zu hemmen vermag. Weiterhin scheint die niedrige Temperatur die enzymatischen Vorgänge, die den Schaden an den Lamellen verursachen, zu verlangsamen und die Zerstörung der Basalmembran zu verhindern. Effektiv ist die Kältetherapie dann, wenn das Pferd für mindestens 24 Stunden mit beiden Vordergliedmaßen (von der Röhre abwärts) in Eiswasser verbracht wird. Sie stellt eine effektive und preiswerte Therapieform dar, die gerade im Anfangsstadium hilft, das Ausmaß der Hufrehe einzudämmen. Diese Maßnahme erweist sich jedoch nur dann als effektiv, wenn der Kühlungseffekt kontinuierlich und bei konstanter niedriger Temperatur gewährleistet wird, was oft schwer umzusetzen ist. Coolpacks, die um die distale Gliedmaße gewickelt werden, sind auch möglich, müssen aber ebenfalls mindestens jede Stunde gewechselt werden, um den Kühlungseffekt beizubehalten. Leider ist es beim Auftreten der ersten sichtbaren Rehesymptome (akutes Stadium) meistens schon zu spät.

Wenn wegen einer Endotoxämie, Metritis, Nachgeburtsverhaltung oder eines Kohlenhydratüberschuss mit der Ausbildung einer Hufrehe zu rechnen ist, sollte sogleich eine prophylaktische Therapie noch vor dem Auftreten erster Symptome eingeleitet werden. Die Einwirkung von Hitze auf den Huf oder die Applikation vasodilatierender Pharmaka wie Acepromazin sollte in der akuten Phase vermieden werden. Die bisherige Annahme, dass eine Vasodilatation die Reparaturmechanismen von strukturellen Schäden zu unterstützen vermag, stellen neuere Untersuchungen infrage: Sie kommen zu dem Ergebnis, dass der Gebrauch von etwa 
Acetylcystein oder Acepromazin keinen Effekt erzielen.

Steht eine Endotoxämie ursächlich im Vordergrund, sollte Flunixin-Meglumin eingesetzt werden. Die zeitgleiche Anwendung von

- Flunixin-Meglumin in der niedrigen Dosierung: 0,25 mg/kg KGW $3 \times$ tgl. und

> Phenylbutazon: $2,2 \mathrm{mg} / \mathrm{kg} 2 \times \mathrm{tgl}$.

kann in diesem Fall sinnvoll sein. FlunixinMeglumin fungiert als Toxinfänger und
Phenylbutazon hat eine stärkere entzündungshemmende Wirkung.

Es existiert eine gute Korrelation zwischen den mikroskopisch sichtbaren Schäden der lamellären Verbindungen und dem klinischen Grad der Lahmheit. Dies bedeutet, dass bei den ersten Anzeichen von Schmerzen durch Hufrehe der Zerstörungsprozess der Blättchen begonnen hat. Jeder zusätzliche mechanische Stress auf die vorgeschädigte Struktur vergrößert den Scha- den und ist kontraindiziert. Somit sollten auch grundsätzlich Leitungsanästhesien bei akutem Reheverdacht unterbleiben, da das Beheben der Schmerzen zur stärkeren Belastung der Gliedmaße führt.

Eine mechanische Unterstützung des Hufes und die Entlastung der tiefen Beugesehne sowie die Unterstützung des Strahls mit schwebender Zehe vervollständigen die Maßnahmen der Rehetherapie. In unserem Haus wird hierfür eine einfache 


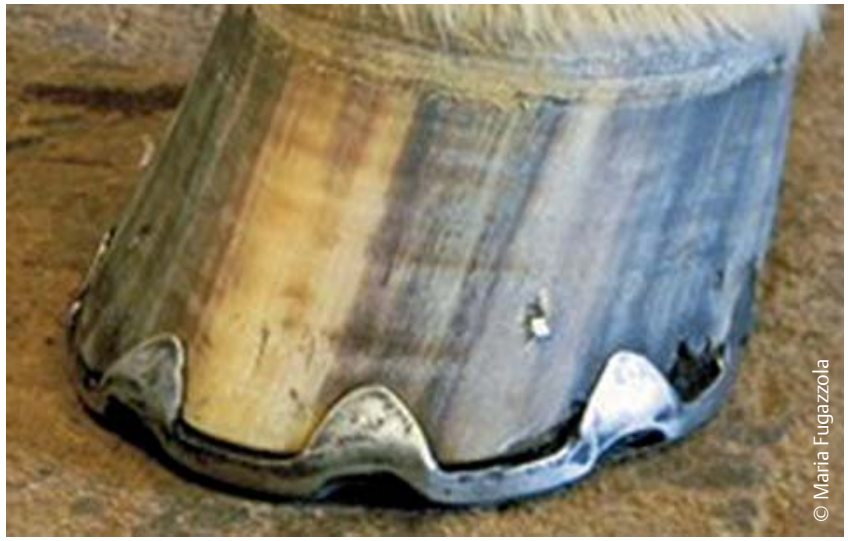

und kostengünstige Lösung mit dem Aufrollen und dem Verwenden von auf das entsprechende Maß zugeschnittenen Mullbinden genutzt. Auch das mechanische Entlasten der betroffenen Gliedmaßen durch das Verbringen des Pferdes in einen Swinglifter ( $\mathbf{A} \mathbf{b} \mathbf{b} . \mathbf{5}$ ) entlastet in der akuten und post-akuten Phase den geschädigten Aufhängemechanismus der Hufbeine.

\section{Prognose}

Trotz frühzeitiger Diagnose und intensiver Therapie ist der destruktive Mechanismus der Rehe oft nicht aufzuhalten. Darauf sollten Besitzer von Anfang an hingewiesen werden. Anhaltspunkte für eine Langzeitprognose geben die Röntgenaufnahmen der ersten 4 Wochen nach dem akuten Schub und die klinische Symptomatik. Ein kontinuierliches Absenken des Hufbeins in der Hufkapsel und eine Rotation über $13^{\circ}$ machen die Prognose ungünstig.

\section{Hufbeinfrakturen}

Die Hufbeinfraktur stellt bei Sportpferden eine relativ häufige Ursache für eine akute Lahmheit dar. Sie ist meistens Konsequenz eines starken Aufschlags des Hufes auf den Boden oder eines Tretens gegen ein unbewegliches Objekt.

Frakturen des Proc. extensorius haben meist einen chronischen Verlauf. Sie können sich in einer nur moderaten Lahmheit oder aber, wenn Hufbeinkörper und Hufbeinäste betroffen sind, auch in einer typischen, hochgradigen Lahmheit äußern.

Bei Hufbeinastfrakturen ist oft eine starke Belastung der seitlichen Hufwand erkennbar. Das Pferd zeigt auf dem betroffenen Bein eine mitunter starke Innenbogen-
Abb. 7 Eisen mit multiplen Zehen- und Seitenkappen als Therapie eines Hufbeinbruchs.

lahmheit. Oft ist dieser Unterschied so eindeutig, dass auf die Leitungsanästhesie verzichtet werden kann und zunächst ein Röntgenbild angefertigt werden sollte.

\section{Röntgen}

Eine lateromediale und eine schräge $\mathrm{Ox}$ spring-Aufnahme führen oft schon zur Diagnose. Der Huf sollte zuvor sehr gründlich gesäubert und ausgeschnitten werden, um Artefakte auf dem Röntgenbild zu vermeiden. Vor allem die Strahlfurchen und Eckstreben sollten mit luftdichtem Material ausgestopft werden, um Luftartefakte in diesen Bereichen zu vermeiden. Die meisten Hufbeinastfrakturen mit Gelenkbeteiligung können in den schrägen Oxspring-Aufnahmen erkannt werden ( $\triangleright$ Abb. 6). Frakturen ohne Gelenkbeteiligung sind dagegen eher in der lateromedialen Aufnahme zu sehen.

\section{Therapie}

Die konservative Therapie für nicht artikuläre und für nicht dislozierte artikuläre Hufbeinastfrakturen verspricht eine gute Ausheilung. Der Heilungserfolg lässt sich röntgenologisch zu Beginn oft nicht darstellen, da es zunächst zu einer bindegewebigen Heilung des Frakturspalts kommt. Mit ihr kann auch das Verbessern der klinischen Symptome, trotz noch sichtbarer Frakturlinie nach einigen Monaten erklärt werden. Bei dem betroffenen Huf müssen der Hufbeinmechanismus und der dadurch ausgelöste Stress auf das Hufbein vorübergehend ausgeschaltet werden. Hierfür können ein geschlossenes Eisen mit seitlichen Kappen ( Abb. 7) oder ein Hufgips angebracht werden. Nach ca. 3 Monaten Boxenruhe, abhängig vom klinischen Erscheinungsbild, kann das Pferd wieder antrainiert werden, es sollte aber weiterhin ein geschlossenes Eisen tragen.

Beim Wechsel auf einen normalen Beschlag muss das Training zunächst wieder reduziert werden, um der Hufkapsel und dem Hufbein Zeit zu geben, sich an die neuen Erschütterungskräfte zu adaptieren.

\section{Prognose}

Bei nicht dislozierten Sagittalfrakturen des Hufbeins ist die Prognose bei jüngeren Pferden günstig. Für Pferde, die älter als 3 Jahre sind, ist sie jedoch als vorsichtiger zu bewerten. Oftmals ist hier die Fixierung mit einer Zugschraube indiziert.

Bei dislozierten Frakturen hängt die Prognose vom Grad der Dislokation im Hufgelenk ab. Eine Heilung nach Osteosynthese kann bei diesen Fällen bis zu 12 Monate dauern.

\section{Fazit}

Bei einer akuten hochgradigen Lahmheit sollte immer erst die gründliche klinische Untersuchung mit detaillierter Anamnese im Vordergrund stehen. Auf besondere Charakteristiken des Bewegungsmusters des lahmen Pferdes ist außerdem besonders zu achten. Eine gute Lahmheitsuntersuchung hat dabei zum Ziel, so genau als möglich den Sitz der Lahmheit zu eruieren und das, ohne dabei vorschnell weiterführende Diagnostika anzuwenden. Neben einer gründlichen Untersuchung muss eine umfassende Aufklärung des Besitzers über das, was letztlich vor Ort möglich ist und welches Restrisiko bestehen bleibt, erfolgen. Auch sollte immer darauf hingewiesen werden, dass in der Außenpraxis eine nur eingeschränkte Diagnostik möglich ist. Diese Aufklärung ist, ebenso wie die Befunddokumentation gleichwohl schriftlich festzuhalten.

\section{Online}

https://doi.org/10.1055/s-0043-110745

\section{Dr. Maria Fugazzola, Dipl. ECVS}

Pferdeklinik Burg Mueggenhausen $\mathrm{GmbH}$

53919 Weilerswist

Fugazzola@pferde-klinik.de 